\title{
Light Harvesting and Efficient Energy Transfer in a Boron-dipyrrin (BODIPY) Functionalized Perylenediimide Derivative
}

\author{
M. Deniz Yilmaz, ${ }^{\dagger}$ O. Altan Bozdemir ${ }^{\ddagger}$ and Engin U. Akkaya* ${ }^{\dagger}$ \\ 'Department of Chemistry, Middle East Technical University, Ankara,Turkey, TR-06531. \\ ${ }^{*}$ Department of Chemistry, Akdeniz University, Antalya, Turkey, TR-07058.
}




\section{Supporting Information}

\section{EXPERIMENTAL PROCEDURES}

\section{General}

All chemicals and solvents purchased from Aldrich were used without further purification. ${ }^{1} \mathrm{H}$ NMR and ${ }^{13} \mathrm{C}$ NMR spectra were recorded using a Bruker DPX-400 in $\mathrm{CDCl}_{8}$ or DMSO- $\mathrm{d}_{6}$ with TMS as internal reference. Absorption spectrometry was performed using a Varian spectrophotometer. Fluorescence measurements were conducted using a Varian Eclipse spectrofluorometer. Column chromatography of all products was performed using Merck Silica Gel 60 (particle size: $0.040-0.063 \mathrm{~mm}, 230-400$ mesh ASTM). Reactions were monitored by thin layer chromatography using fluorescent coated aluminum sheets. Solvents used for spectroscopy experiments were spectrophotometric grade. Mass spectrometry measurements were done at the Ohio State University Mass Spectrometry and Proteomics Facility, Ohio, U.S.A., Kent Mass Spectrometry Laboratory, Kent, U.K., or Hacettepe University, Mass Spectrometry Facility, Ankara, Turkey.

\section{Synthesis}

\section{4-(3-azidopropoxy)benzaldehyde ( 1 ):}

A mixture of 4-(3-bromopropoxy)benzaldehyde (5.37 mmol, $1.3 \mathrm{~g}$ ) and $\mathrm{NaN}_{3}$ (13.425 mmol, $0.87 \mathrm{~g}$ ) in $20 \mathrm{ml} \mathrm{DMF}$ was heated at $100{ }^{\circ} \mathrm{C}$ overnight. The mixture was poured to water and extracted with $\mathrm{CHCl}_{3}$ several times. Removal of solvent gave the pure azide compound. Colorless oil (1.05 g, $99 \%)$

${ }^{1} \mathrm{H}$ NMR (400 MHz, CDCl3) $\delta 9.85$ (s, 1H), 7.81 (d, J=7.75 Hz, 2H), 6.98 (d, J=7.0 Hz, 2H), $4.17(\mathrm{t}, J=4.0 \mathrm{~Hz}, 2 \mathrm{H}), 3.58(\mathrm{t}, J=3.5 \mathrm{~Hz}, 2 \mathrm{H}), 2.03(\mathrm{tt}, J=2.0 \mathrm{~Hz}, 2 \mathrm{H}) .{ }^{13} \mathrm{C} \mathrm{NMR}(100 \mathrm{MHz}$, $\left.\mathrm{CDCl}_{3}\right) \delta 190.6,163.7,131.9,130.0,114.7,64.9,48.0,28.5$. Elemental analysis: Found: C, 59.33; H, 5.49; N, $20.39 \mathrm{C}_{10} \mathrm{H}_{11} \mathrm{~N}_{3} \mathrm{O}_{2}$ requires C, 58.53; H, 5.40; N, 20.48. 


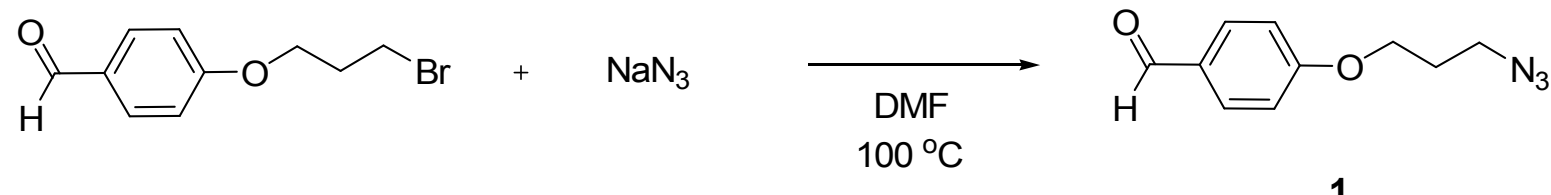

\section{4,4-difluoro-8-(4-(3-azidopropoxy))phenyl-1,3,5,7-tetramethyl-2,6-diethyl-4-bora-3a,4a- diaza-s-indacene ( 3 ):}

3-ethyl-2,4-dimethyl pyrrole (5.26 mmol, $648 \mathrm{mg}$ ) and compound 1 ( $2.6 \mathrm{mmol}, 533 \mathrm{mg}$ ) were dissolved in $200 \mathrm{ml}$ absolute $\mathrm{CH}_{2} \mathrm{Cl}_{2}$ ( argon gas was bubbled through $\mathrm{CH}_{2} \mathrm{Cl}_{2}$ for $30 \mathrm{~min}$ ) under argon atmosphere. One drop of TFA was added and the solution stirred at room temperature nearly 3-4 $\mathrm{h}$. At this point, a solution of DDQ $(2.6 \mathrm{mmol}, 640 \mathrm{mg})$ in $50 \mathrm{ml}$ absolute $\mathrm{CH}_{2} \mathrm{Cl}_{2}$ was added, stirring was continued for 30 min followed by the addition of $3 \mathrm{ml}$ $\mathrm{Et}_{3} \mathrm{~N}$ and $3 \mathrm{ml} \mathrm{BF}_{3}$. $\mathrm{OEt}_{2}$. After stirring $30 \mathrm{~min}$. the reaction mixture was washed three times with water and dried over $\mathrm{Na}_{2} \mathrm{SO}_{4}$. The solvent was evaporated and the residue was purified by silica gel column chromatography $\left(\mathrm{CHCl}_{3}\right)$. Red solid (374 mg, 30\%)

${ }^{1} \mathrm{H}$ NMR $\left(400 \mathrm{MHz}, \mathrm{CDCl}_{3}\right) \delta 7.10(\mathrm{~d}, J=7.0 \mathrm{~Hz}, 2 \mathrm{H}), 6.93(\mathrm{~d}, J=7.0 \mathrm{~Hz}, 2 \mathrm{H}), 4.05$ (t, $J=4.0$ $\mathrm{Hz}, 2 \mathrm{H}), 3.49$ (t, $J=3.5 \mathrm{~Hz}, 2 \mathrm{H}), 2.45$ (s, 6H), 2.25 (q, $J=2.23 \mathrm{~Hz}, 4 \mathrm{H}), 2.01$ (tt, $J=2.0 \mathrm{~Hz}, 2 \mathrm{H}$ ), 1.27 (s, 6H), $0.91(\mathrm{t}, J=0.9 \mathrm{~Hz}, 6 \mathrm{H}){ }^{13} \mathrm{C}$ NMR $\left(100 \mathrm{MHz}, \mathrm{CDCl}_{3}\right) \delta$ 158.6, 153.1, 139.7, 137.9, 132.2, 130.7, 129.1, 127.7, 114.5, 64.2, 47.8, 28.3, 16.6, 14.1, 12.0, 11.3. Elemental analysis: Found: $\mathrm{C}, 65.44 ; \mathrm{H}, 6.91 ; \mathrm{N}, 14.53 \mathrm{C}_{26} \mathrm{H}_{32} \mathrm{BF}_{2} \mathrm{~N}_{5} \mathrm{O}$ requires $\mathrm{C}, 65.14 ; \mathrm{H}, 6.73 ; \mathrm{N}, 14.61$. 


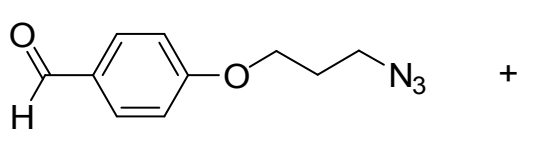

1

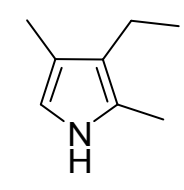

2
1. TFA, DDQ

2. $\mathrm{Et}_{3} \mathrm{~N}, \mathrm{BF}_{3} \cdot \mathrm{OEt}_{2}$

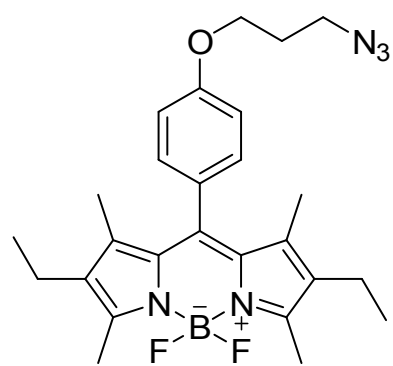

3

N,N'-Dicyclohexyl-1,6,7,12-tetra(4-propargyloxyphenoxy)perylene-3,4:9,10tetracarboxylic diimide ( 6 ):

A mixture of N,N'-Dicyclohexyl-1,6,7,12-tetrachloroperylene-3,4,:9,10-tetracarboxylic Acid Diimide (0.72 mmol, $500 \mathrm{mg})$, 4-propargyloxyphenol (6.75 mmol, $1 \mathrm{~g})$ and $\mathrm{K}_{2} \mathrm{CO}_{3}(7.25$ mmol, $1 \mathrm{~g}$ ) in $20 \mathrm{ml} \mathrm{NMP}$ was heated at $90{ }^{\circ} \mathrm{C}$ overnight. A cooled mixture was poured into 20 $\mathrm{ml} 10 \% \mathrm{HCl}$ solution and filtered by suction, washed three times with water. The resulting precipitate was purified by column chromatography on silica gel (first $\mathrm{CHCl}_{3}$, then $\left.\mathrm{CHCl}_{3} / \mathrm{Hexane}(5: 1)\right)$. Red solid (410 mg, 50\%).

${ }^{1} \mathrm{H}$ NMR (400 MHz, $\left.\mathrm{CDCl}_{3}\right) \delta 8.01$ (s, 4H), $6.79(\mathrm{~s}, 16 \mathrm{H}), 4.80(\mathrm{~m}, 2 \mathrm{H}), 4.62(\mathrm{~s}, 8 \mathrm{H}), 2.52$ (s, 4H), 1.03-2.01 (m, 20H) ${ }^{13} \mathrm{C}$ NMR (100 MHz, $\left.\mathrm{CDCl}_{3}\right) \delta 163.7,156.3,154.5,149.5,132.8$, 123.0, 121.2, 119.9, 119.4, 119.2, 116.4, 78.5, 75.7, 56.4, 54.0, 29.1, 26.5, 25.4. Elemental analysis: Found: C, 75.69; H, 4.68; N, $2.34 \mathrm{C}_{72} \mathrm{H}_{54} \mathrm{~N}_{2} \mathrm{O}_{2}$ requires C, 75.91; H, 4.78; N, 2.46. MS (MALDI) $1138.4\left(\mathrm{M}^{+}\right)$. 


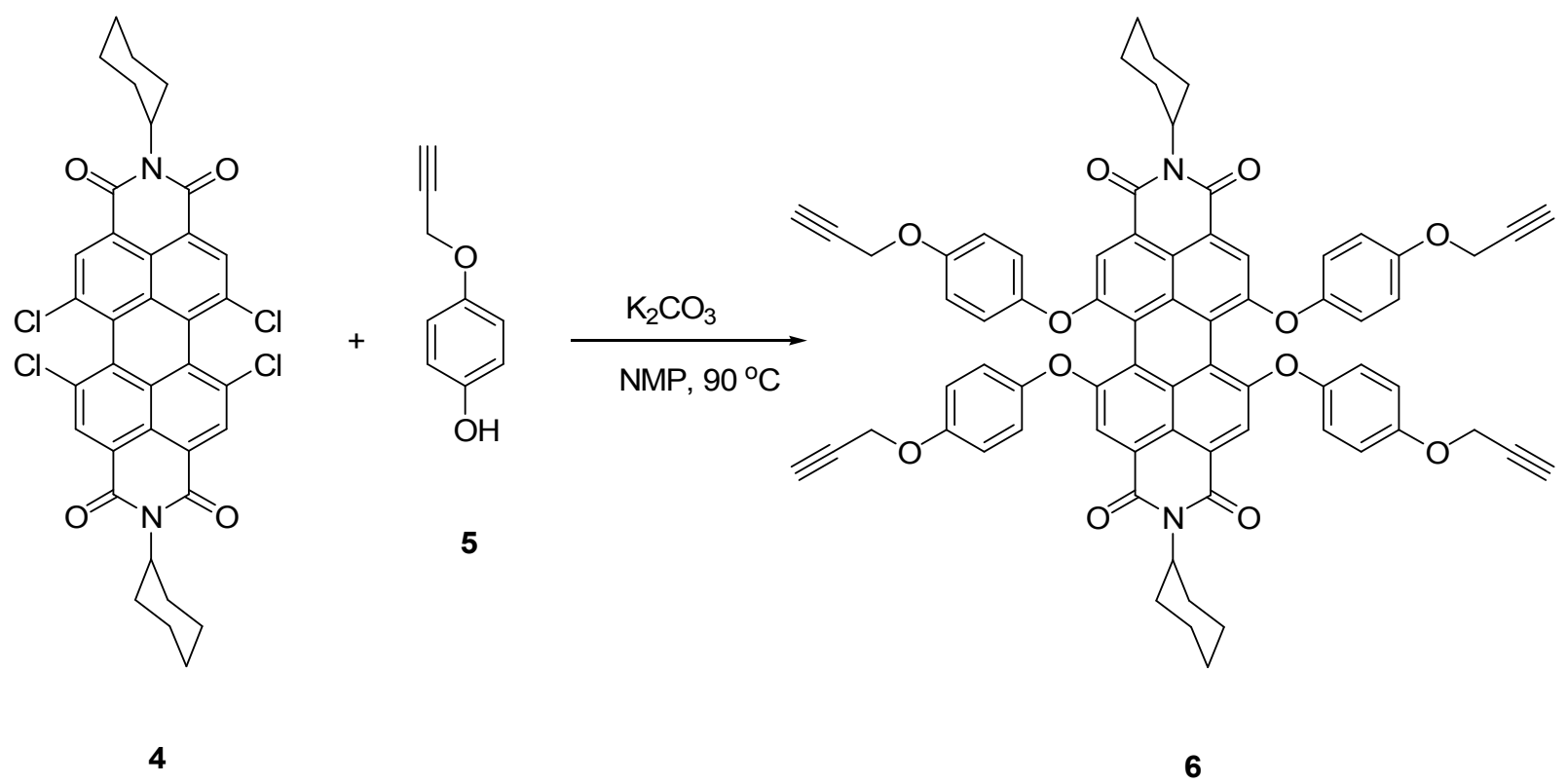

\section{Synthesis of compound 7 :}

A solution of the compound $3(0.225 \mathrm{mmol}, 107.8 \mathrm{mg})$, compound 6 (0.0535 $\mathrm{mmol}, 61 \mathrm{mg})$, sodium ascorbate $(0.0225 \mathrm{mmol}, 4.46 \mathrm{mg})$, and $\mathrm{CuSO}_{4}(0.01125 \mathrm{mmol}, 2.8 \mathrm{mg})$ in a 12:1:1 mixture of $\mathrm{CHCl}_{3}$, EtOH and water $(14 \mathrm{ml})$ was stirred at room temperature for $72 \mathrm{~h}$. After evaporation of the solvents, the crude product was purified by column chromatography $\left(\mathrm{CHCl}_{3}\right)$. Red solid (130.9 mg, 80\%)

${ }^{1} \mathrm{H}$ NMR $\left(400 \mathrm{MHz}, \mathrm{CDCl}_{3}\right) \delta 8.02(\mathrm{~s}, 4 \mathrm{H}), 7.65(\mathrm{~s}, 4 \mathrm{H}), 7.11(\mathrm{~d}, J=7.1 \mathrm{~Hz}, 8 \mathrm{H}), 6.89(\mathrm{~d}, J=$ $6.9 \mathrm{~Hz}, 8 \mathrm{H}), 6.82(\mathrm{~s}, 16 \mathrm{H}), 5.11(\mathrm{~s}, 8 \mathrm{H}), 4.82(\mathrm{~m}, 2 \mathrm{H}), 4.64(\mathrm{t}, J=4.6 \mathrm{~Hz}, 8 \mathrm{H}), 4.02(\mathrm{t}, J=4.0$ $\mathrm{Hz}, 8 \mathrm{H}), 2.44(\mathrm{~s}, 24 \mathrm{H}), 2.18(\mathrm{q}, J=2.2 \mathrm{~Hz}, 16 \mathrm{H}), 1.03-2.05(\mathrm{~m}, 52 \mathrm{H}), 0.92(\mathrm{t}, 24 \mathrm{H}){ }^{13} \mathrm{C}$ NMR $\left(100 \mathrm{MHz} \mathrm{CDCl}_{3}\right) \delta 163.7,158.9,156.4,155.3,153.6,149.2,144.1,140.0,138.3,132.7$, 131.2, 129.7, 128.5, 123.2, 123.0, 121.4, 119.8, 119.0, 117.2, 116.2, 115.0, 113.3, 107.5, 106.0, 64.4, 62.6, 47.3, 30.0, 29.1, 26.5, 21.8, 17.0, 14.6, 12.5, 11.9. MS (MALDI) 3036.4 (M-F) ${ }^{+}$. 


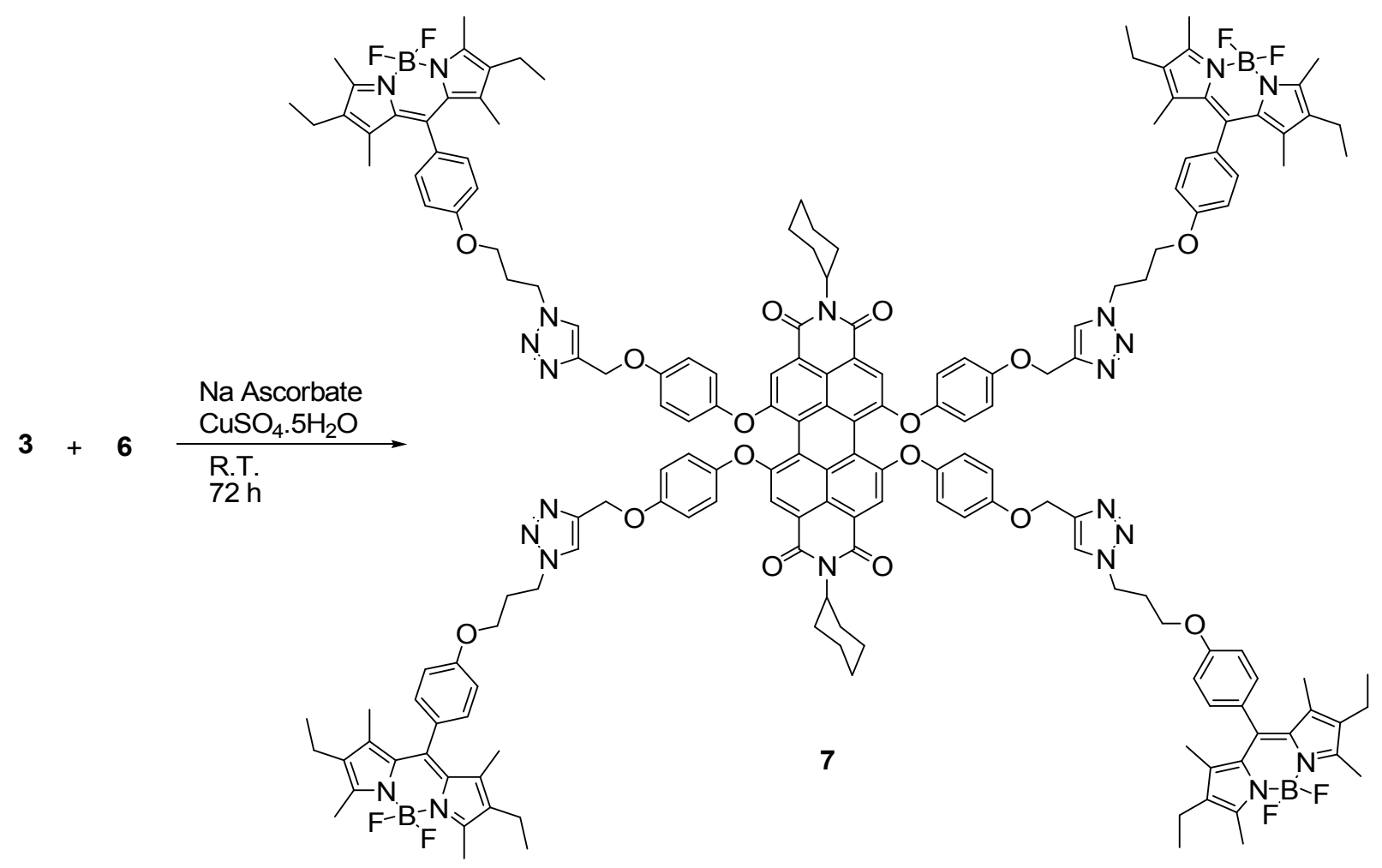


TABLE S1. Selected spectral data for compounds 3, 6 and 7.

\begin{tabular}{|c|c|c|c|c|c|}
\hline \multirow{2}{*}{ Compounds } & \multirow{2}{*}{ Absorption $\lambda(\mathrm{nm})\left(\varepsilon, \mathbf{M}^{-1} \mathrm{~cm}^{-1}\right)$} & \multicolumn{4}{|c|}{ Emission at RT } \\
\hline & & $\lambda_{\text {bodipy }}(\mathbf{n m})$ & $\lambda_{\text {per }}(\mathbf{n m})$ & $\Phi_{\text {bodipy }}$ & $\Phi_{\text {per }}$ \\
\hline 3 & $526(62000)$ & 538 & & 0.85 & \\
\hline 6 & $450,540,582(45700)$ & & 616 & & 0.27 \\
\hline 7 & $526(240000), 582(45000)$ & 538 & 616 & 0.01 & 0.60 \\
\hline
\end{tabular}


Figure S1: ${ }^{1} \mathrm{H}$ and ${ }^{13} \mathrm{C}$ NMR spectrum of compound 1
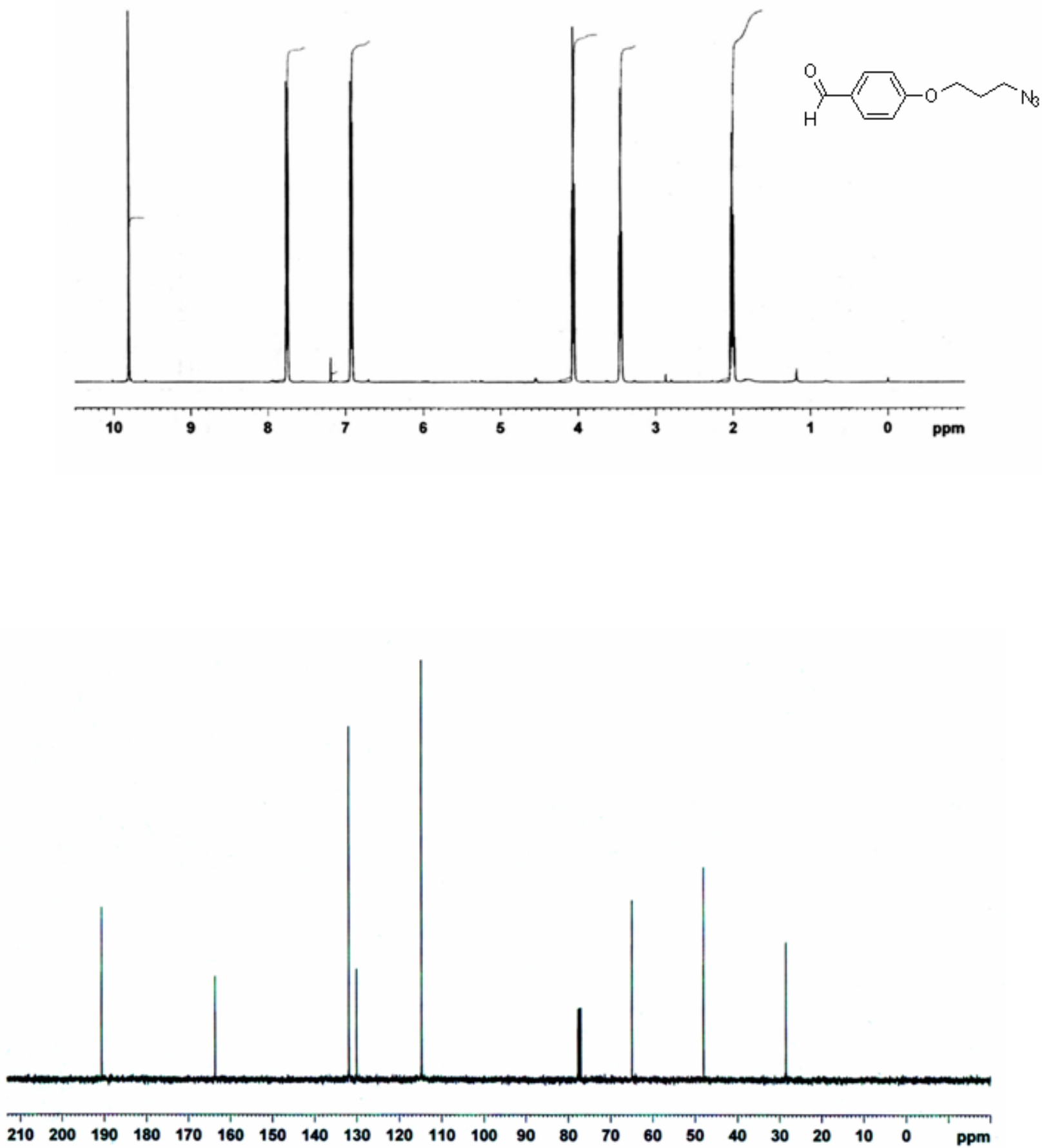
Figure S2: ${ }^{1} \mathrm{H}$ and ${ }^{13} \mathrm{C}$ NMR spectrum of compound 3
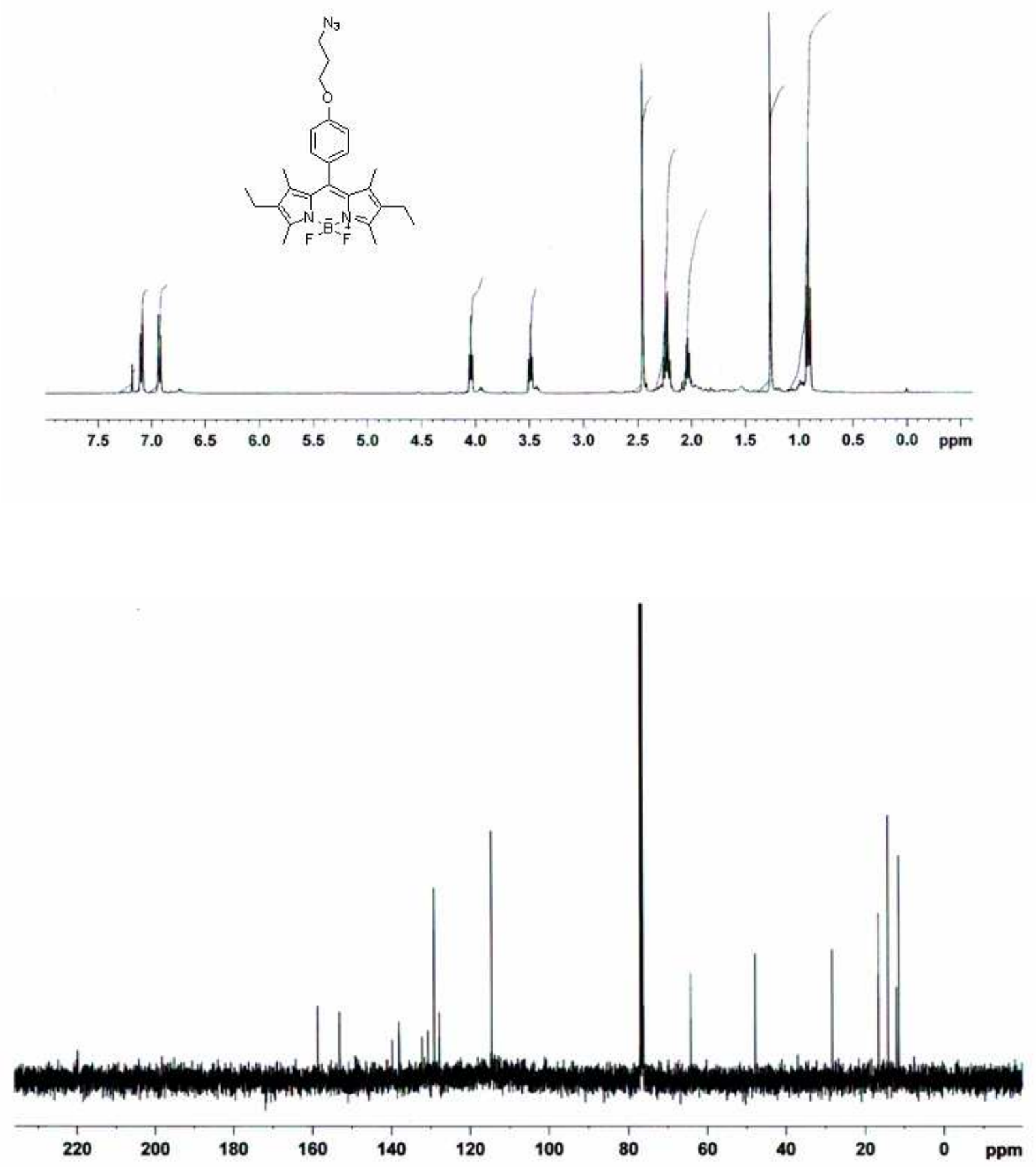
Figure S3: ${ }^{1} \mathrm{H}$ and ${ }^{13} \mathrm{C}$ NMR spectrum of compound 6
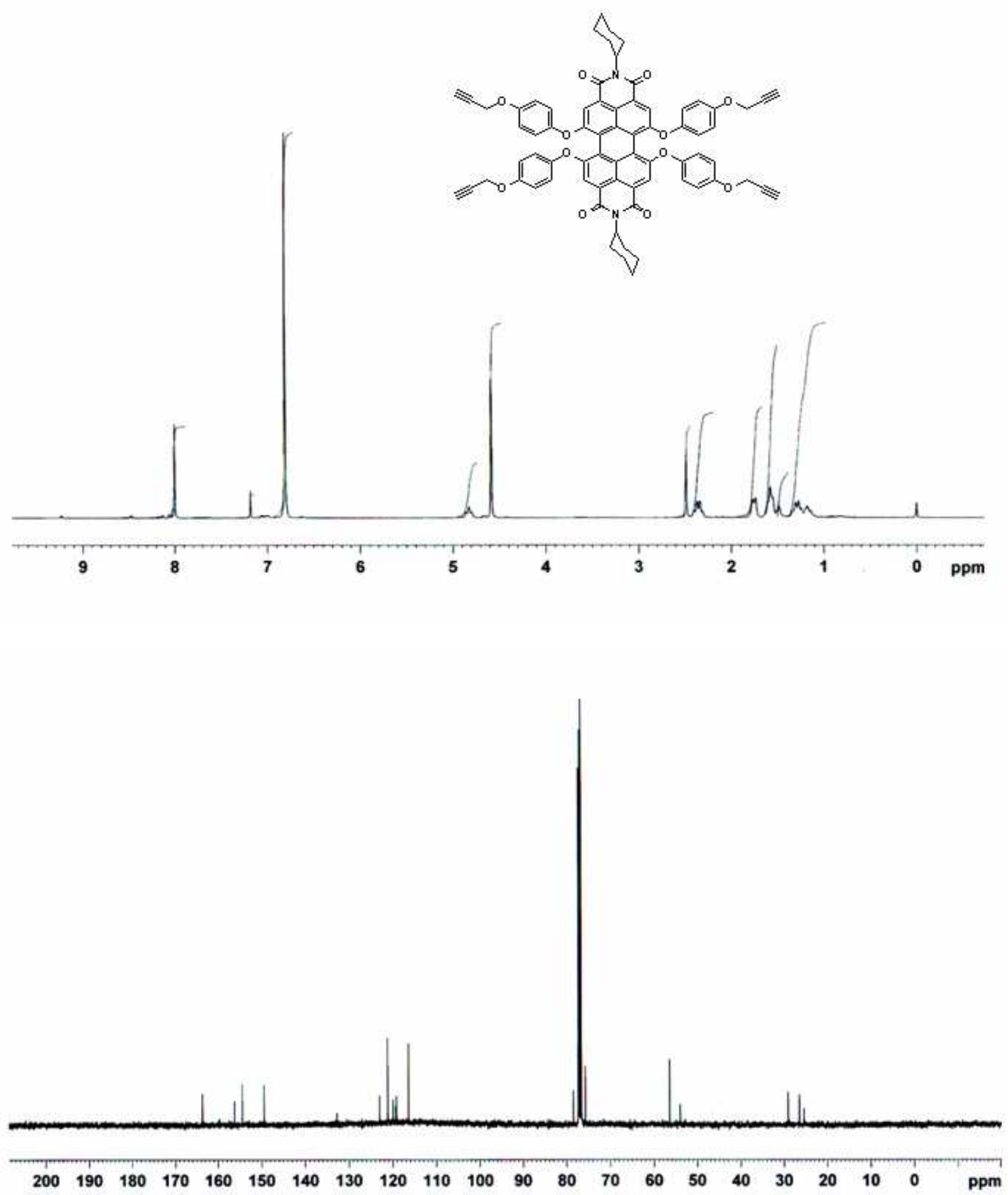
Figure S4: ${ }^{1} \mathrm{H}$ and ${ }^{13} \mathrm{C}$ NMR spectrum of compound 7
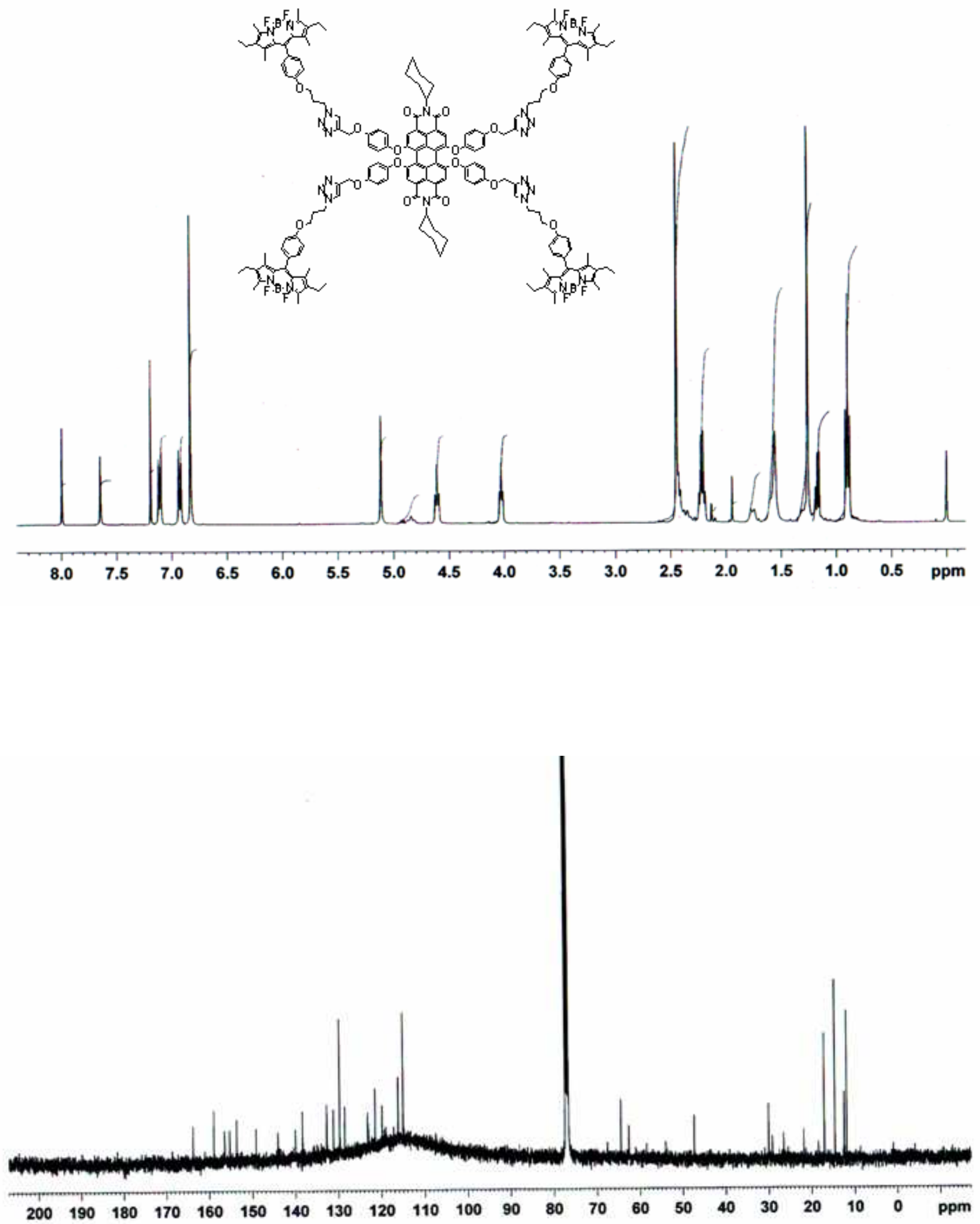